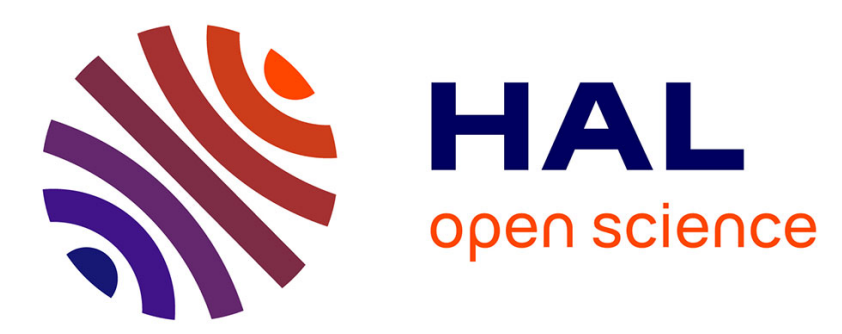

\title{
Modelling and numerical simulation of plasma flows with two-fluid mixing
}

Remi Sentis, Didier Paillard, Céline Baranger, Patricia Seytor

\section{To cite this version:}

Remi Sentis, Didier Paillard, Céline Baranger, Patricia Seytor. Modelling and numerical simulation of plasma flows with two-fluid mixing. 2010. hal-00484090

\author{
HAL Id: hal-00484090 \\ https://hal.science/hal-00484090 \\ Preprint submitted on 17 May 2010
}

HAL is a multi-disciplinary open access archive for the deposit and dissemination of scientific research documents, whether they are published or not. The documents may come from teaching and research institutions in France or abroad, or from public or private research centers.
L'archive ouverte pluridisciplinaire HAL, est destinée au dépôt et à la diffusion de documents scientifiques de niveau recherche, publiés ou non, émanant des établissements d'enseignement et de recherche français ou étrangers, des laboratoires publics ou privés. 


\title{
Modelling and numerical simulation of plasma flows with two-fluid mixing
}

\author{
Rémi Sentis ${ }^{1}$, Didier Paillard, Céline Baranger ${ }^{2}$, Patricia Seytor \\ CEA, DAM, DIF, 91297 Arpajon, France
}

\begin{abstract}
For the modelling of plasma flows at very high temperature such the ones produced by laser beams, one must account for a bi-temperature compressible Euler system coupled to electron thermal conduction and radiative conduction. Moreover, mixing of two different fluids can occur, the two fluids occupying the same volume. For modelling such a phenomenon, instead of dealing with the conservation of mass, momentum and energy for each fluid, we propose here a simplified model which will be easier to implement in a multi-physics Lagrangian 2D code. The principle is to use a closure for expressing the relative velocity between the two fluids with the help of the gradient of the concentration. So, besides the classical system, the final model consists in a non-linear diffusion equation for the concentration and an equation for the mixing kinetic energy (analogous to the one used in turbulence models). We present also first numerical 2D simulations using this model.
\end{abstract}

Keywords. Mixing model. Multi-material two-temperature Euler system. Concentration diffusion equation. Mixing kinetic energy equation.

Introduction. In the multicomponent plasma flows at very high temperature (for instance in the plasma produced by laser beams), mixing of two different fluids can occur. In the physical interesting cases, the mixing phenomena occur in narrow regions of the simulation domain, where the relative velocity between the two fluids is large if compared to the thermal one. A classical model for such phenomena consists in a system of six equations which corresponds to the conservation of mass, momentum and energy for each fluid. The two fluids are assumed to occupy the same volume (the global pressure is the sum of the pressures of the two fluids). The aim of this work is to perform numerical simulations dealing with this mixing problem ; this has been addressed by numerous earlier works, see [6], [7], [8], [10].

Here, our aim is to perform global two-dimension simulations taking into account the mixing phenomena and the coupling with an electron temperature equation (with thermal conduction). The related theoretical model has been written for instance in [3]: it consists of the previous system of six equations with an evolution equation for the electron temperature. Recall that in the single species case, the simplest model is the so-called two-temperature Euler system which reads as follows (see for instance [2],[9])

\footnotetext{
${ }^{1}$ corresponding author, remi.sentis@cea.fr

${ }^{2}$ permanent address : CEA, DAM, CESTA, 33114 Le Barp, France
} 


$$
\begin{aligned}
\frac{\partial}{\partial t} \rho+\nabla \cdot(\rho \mathbf{U}) & =0, \\
\frac{\partial}{\partial t}(\rho \mathbf{U})+\nabla \cdot(\rho \mathbf{U} \mathbf{U})+\nabla\left(P_{0}+P_{e}\right) & =0, \\
\frac{\partial}{\partial t} \mathcal{E}_{0}+\nabla \cdot\left(\mathbf{U} \mathcal{E}_{0}\right)+\nabla \cdot\left(\mathbf{U} P_{0}\right)+\mathbf{U} \cdot \nabla P_{e} & =\Upsilon_{0}, \\
\frac{\partial}{\partial t} \mathcal{E}_{e}+\nabla \cdot\left(\mathbf{U} \mathcal{E}_{e}\right)+P_{e} \nabla \cdot \mathbf{U} & =-\Upsilon_{0} .
\end{aligned}
$$

where we denote $\rho$ the density, $\mathbf{U}$ the velocity, $\mathcal{E}_{e}$ the electron energy and $\mathcal{E}_{0}$ the total ion energy (kinetic plus internal energy). Moreover, $\Upsilon_{0}$ denotes a relaxation term between the ion and electron temperatures. Notice that other physical phenomena are to be taken into account such as the electron thermal conduction, a radiative transfer model and a laser energy deposition model. All these phenomena correspond to added terms in the evolution equation for $\mathcal{E}_{e}$ which are coupled to other equations. This leads to heavy complex simulations, in particular in a multi-dimension framework, which are generally performed using Lagrangian codes, see for instance FCI2 [1], [4]. For all these reasons, reduced models in which the mixing process is roughly simulated are very usefull. This paper is aiming at proposing such a reduced model which may be easily implemented in an existing Lagrangian code.

In the first section we recall the classical model for mixing phenomena which consists in a system of three equations for each fluid (conservation of mass, momentum and energy), besides an equation for the electron energy, as it is written in the reference paper [3]. In the second section, we emphasize the closures for getting a five-equation model which consists in the conservation equations of mass, momentum and energy for the averaged fluid coupled with an equation of concentration and an equation for the mixing kinetic energy. These closures are made under the assumption that the friction coefficient becomes large. The relative velocity is given by a closure using the gradient of the concentration ; it allows to state a diffusion approximation model for the concentration (it looks like a diffusion model for combustion or turbulence problem, see [14]). In the second part of this section we introduce the supplementary terms due to the coupling with the electron energy balance equation. In a certain way, our model is in the same family of one of the mixing models introduced in [6] or [15].

In the last section, we give some enlightenments on the numerical methods for solving this mixing model. Some preliminary numerical results are also presented firstly in a one-dimension toy problem and secondly in a two-dimension problem where we account for electron temperature coupling as well as a simple laser energy deposition model.

We thank sincerly Gilles Carré for his work on the implemention of the numerical method and his help for tuning the numerical code.

\section{Initial model for mixing of plasmas}

We recall here the detailed model given in [3] (see particularly $§ \S 1.4,1.5)$. In the sequel, we do not account for the ion thermal conduction flux and the thermo-electric terms, for the sake of simplicity. We first write this model without the coupling between the electron and ion temperatures for focusing on its main features. The coupling between the electron and ion will be introduced in the second subsection.

\subsection{Model without the coupling with the electron temperature}

For each fluid ( $a$ and $b$ ) denote by $\rho_{a}, \rho_{b}$ the densities, $\mathbf{u}_{a}, \mathbf{u}_{b}$ the velocities and by $E_{a}, E_{b}$ the total specific energies. So $\varepsilon_{a}=E_{a}-\frac{1}{2}\left|\mathbf{u}_{a}\right|^{2}$ and $\varepsilon_{b}=E_{b}-\frac{1}{2}\left|\mathbf{u}_{b}\right|^{2}$ are the specific internal energies. The initial model is the following (with $q=a$ or $b$ ) 


$$
\begin{aligned}
\frac{\partial}{\partial t} \rho_{q}+\nabla \cdot\left(\rho_{q} \mathbf{u}_{q}\right) & =0, \\
\frac{\partial}{\partial t}\left(\rho_{q} \mathbf{u}_{q}\right)+\nabla \cdot\left(\rho_{q} \mathbf{u}_{q} \mathbf{u}_{q}+P_{q}\right) & =\boldsymbol{\Xi}_{q}, \\
\frac{\partial}{\partial t}\left(\rho_{q} E_{q}\right)+\nabla \cdot\left(\rho_{q} \mathbf{u}_{q} E_{q}+\mathbf{u}_{q} P_{q}\right) & =\Omega_{q}
\end{aligned}
$$

knowing that the friction or drag term may read as

$$
\boldsymbol{\Xi}_{a}=-\boldsymbol{\Xi}_{b}=\widetilde{\nu} \rho_{a} \rho_{b}\left(\mathbf{u}_{b}-\mathbf{u}_{a}\right)
$$

(uu denotes as usual a tensor). In the sequel, we assume that each fluid obeys a perfect gas law with a polytropic coefficient $\gamma_{q}$; that is to say we have $P_{q}=\left(\gamma_{q}-1\right) \rho \varepsilon_{q}$.

In [3], the author gives the expression of the relaxation coefficient $\widetilde{\nu}$ which depends on the temperatures of the two species and on the physical characteristics of the particles (and on the relative velocity when it is not small compared with sound speed). On the other hand, we have

$$
\Omega_{a}=-\Omega_{b}=\Omega^{\#}+\widetilde{\nu} \rho_{a} \rho_{b}\left(\mathbf{u}_{b}-\mathbf{u}_{a}\right) \frac{m_{a} \mathbf{u}_{a}+m_{b} \mathbf{u}_{b}}{m_{a}+m_{b}}, \quad \Omega^{\#}=\nu^{\#} \rho_{a} \rho_{b}\left(T_{b}-T_{a}\right)
$$

where $m_{a}, m_{b}$ are the mass of the two ion species and coefficient $\nu^{\#}$ corresponds to the relaxation between the temperatures of the two species.

As we will see in the sequel, the order of magnitude of the coefficient $\widetilde{\nu}$ is crucial in order to determine whether the mixing of the two plasmas can occur or not.

\subsection{Model taking into account the electron temperature}

We account here for the coupling of the previous model with the electron population. Let us denote the electron density, temperature, energy per unit of volume and pressure by $N_{e}, T_{e}, \mathcal{E}_{e}$ and $P_{e}$. Firstly, if $\eta_{a}$ and $\eta_{b}$ are the ionization levels divided by the atomic mass, according to the electric neutrality of the plasma, we get

$$
N_{e}=\eta_{a} \rho_{a}+\eta_{b} \rho_{b}
$$

Notice also that the electron velocity $\mathbf{U}_{e}$ is not equal to the averaged ion velocity but is characterized by

$$
N_{e} \mathbf{U}_{e}=\eta_{a} \rho_{a} \mathbf{u}_{a}+\eta_{b} \rho_{b} \mathbf{u}_{b}
$$

(that is to say the electric current is zero). Secondly, we know that

$$
\mathcal{E}_{e}=\frac{3}{2} N_{e} T_{e}, \quad P_{e}=N_{e} T_{e}
$$

Of course the continuity equation (1) still holds.

The ion momentum and energy balance equations have to be modified to take into account the electron pressure, that is to say a force proportional to $\frac{1}{N_{e}} \nabla P_{e}$ (corresponding to the electrostatic field); so we get

$$
\begin{aligned}
\frac{\partial}{\partial t}\left(\rho_{q} \mathbf{u}_{q}\right)+\nabla \cdot\left(\rho_{q} \mathbf{u}_{q} \mathbf{u}_{q}+P_{q}\right)+\frac{\eta_{q} \rho_{q}}{N_{e}} \nabla P_{e} & =\boldsymbol{\Xi}_{q} \\
\frac{\partial}{\partial t}\left(\rho_{q} E_{q}\right)+\nabla \cdot\left(\rho_{q} \mathbf{u}_{q} E_{q}+\mathbf{u}_{q} P_{q}\right)+\frac{\eta_{q} \rho_{q}}{N_{e}} \mathbf{u}_{q} \cdot \nabla P_{e} & =\Upsilon_{q}+\Omega_{q} .
\end{aligned}
$$

here $\Upsilon_{q}$ denotes a relaxation term between the ion and electron temperatures and reads as

$$
\Upsilon_{a}=\nu_{a}^{E} \rho_{a} N_{e}\left(T_{e}-\varepsilon_{a} / C_{v, a}\right), \quad \Upsilon_{b}=\nu_{b}^{E} \rho_{b} N_{e}\left(T_{e}-\varepsilon_{b} / C_{v, b}\right) .
$$


where $C_{v, q}$ is the heat capacity and $\nu_{q}^{E}$ is a positive coefficient. Let us state now the electron energy balance equation accounting for the electron thermal conduction; so we set $\mathbf{q}_{\mathrm{th}}$ the Spitzer thermal conduction flux equal to $-\kappa\left(T_{e}\right) \nabla T_{e}$ (where $\kappa$ is roughly speaking proportional to $T_{e}^{5 / 2}$ ) and we have

$$
\frac{\partial}{\partial t} \mathcal{E}_{e}+\nabla \cdot\left(\mathcal{E}_{e} \mathbf{U}_{e}\right)+P_{e} \nabla \cdot \mathbf{U}_{e}+\nabla \cdot \mathbf{q}_{\mathrm{th}}=-\left(\Upsilon_{a}+\Upsilon_{b}\right)
$$

Notice that the friction phenomenon between ions and electrons has not been accounted here since it is generally less important than the friction between the two ion species (notice that this is not always true, particularly at the very beginning of the collision process, but it is sufficient here to explain the main ideas of our method).

Of course, one can check easily that the total energy $\mathcal{E}_{e}+\rho_{a} E_{a}+\rho_{b} E_{b}$ satisfies a balance equation

$$
\begin{aligned}
& \frac{\partial}{\partial t}\left(\rho_{a} E_{a}+\rho_{b} E_{b}+\mathcal{E}_{e}\right)+\nabla \cdot \mathbf{q}_{\mathrm{th}}= \\
& -\nabla \cdot\left(\rho_{a} E_{a} \mathbf{u}_{a}+\rho_{b} E_{b} \mathbf{u}_{b}+\mathbf{u}_{a} P_{a}+\mathbf{u}_{b} P_{b}+\mathbf{U}_{e}\left(P_{e}+\mathcal{E}_{e}\right)\right)
\end{aligned}
$$

\section{Reduced model}

For the sake of simplicity, we withdraw in the first sub-section the coupling terms with the electron energy equation. The correction terms accounting this coupling are given in the second sub-section.

\subsection{Model without the coupling with the electron temperature}

Let us define the averaged physical quantities:

$$
\rho=\rho_{a}+\rho_{b}, \quad \mathbf{U}=\left(\rho_{a} \mathbf{u}_{a}+\rho_{b} \mathbf{u}_{b}\right) / \rho
$$

are the mass density and the mean ion velocity

$\mathbf{V}=\mathbf{u}_{a}-\mathbf{u}_{b}$, the relative velocity

$c=\rho_{a} / \rho$, the mass concentration of material $a$ (so we have $\rho(1-c)=\rho_{b}$ )

$P_{0}=P_{a}+P_{b}$, the global ion pressure.

The global specific internal energy $\varepsilon$ is

$$
\varepsilon=\left(\rho_{a} \varepsilon_{a}+\rho_{b} \varepsilon_{b}\right) / \rho=c \varepsilon_{a}+(1-c) \varepsilon_{b}
$$

Let us also denote

$$
K=\frac{1}{2}|\mathbf{V}|^{2} c(1-c)
$$

which is the specific mixing kinetic energy. It is easy to check that the total energy $\mathcal{E}_{0}$ satisfies

$$
\mathcal{E}_{0}=\rho_{a} E_{a}+\rho_{b} E_{b}=\rho \varepsilon+\frac{1}{2} \rho|\mathbf{U}|^{2}+\rho K .
$$

One can show after standard calculus that :

$$
\begin{aligned}
& \frac{\partial}{\partial t} \rho+\nabla \cdot(\rho \mathbf{U})=0, \\
& \frac{\partial}{\partial t}(\rho \mathbf{U})+\nabla \cdot(\rho \mathbf{U} \mathbf{U})+\nabla P_{0}=-\nabla \cdot(\rho \mathbf{V V} c(1-c)), \\
& \frac{\partial}{\partial t}(\rho c)+\nabla \cdot(\rho c \mathbf{U})+\nabla \cdot(\rho c(1-c) \mathbf{V})=0,
\end{aligned}
$$


Moreover, if one defines $\sigma=\rho \widetilde{\nu}$, one may state an equation for the relative velocity

$$
\begin{aligned}
& \frac{\partial}{\partial t} \mathbf{V}+\nabla \cdot(\mathbf{U V})-\nabla \cdot\left(|\mathbf{V}|^{2} \frac{2 c-1}{2}\right)+\mathcal{B}+\mathcal{A}=-\sigma \mathbf{V} \\
& \mathcal{B}=-\nabla \cdot\left(\mathbf{V V} \frac{2 c-1}{2}\right)+\nabla \cdot\left(|\mathbf{V}|^{2} \frac{2 c-1}{2}\right)+\frac{2 c-1}{2}[(\nabla \cdot \mathbf{V}) \mathbf{V}-(\mathbf{V} \cdot \nabla) \mathbf{V}]+(\mathbf{V} \cdot \nabla) \mathbf{U}-(\nabla \cdot \mathbf{U}) \mathbf{V}
\end{aligned}
$$

with

$$
\mathcal{A}=\left(\gamma_{a}-1\right) \frac{1}{\rho_{a}} \nabla\left(\rho_{a} \varepsilon_{a}\right)-\left(\gamma_{b}-1\right) \frac{1}{\rho_{b}} \nabla\left(\rho_{b} \varepsilon_{b}\right)
$$

In the monodimensional case, $\mathcal{B}$ is equal to zero, so in the sequel, we always withdraw $\mathcal{B}$.

Afterwards, the ion total energy balance equation reads as

$$
\frac{\partial}{\partial t} \mathcal{E}_{0}+\nabla \cdot\left(\mathcal{E}_{0} \mathbf{U}\right)+\nabla \cdot\left(P_{0} \mathbf{U}+\rho c(1-c) \mathbf{V G}\right)+\nabla \cdot(2 \rho K \mathbf{U}-(2 c-1) \rho K \mathbf{V})=0
$$

with

$$
\mathcal{G}=\frac{1}{\rho_{a}}\left(P_{a}+\rho_{a} \varepsilon_{a}\right)-\frac{1}{\rho_{b}}\left(P_{b}+\rho_{b} \varepsilon_{b}\right)=\gamma_{a} \varepsilon_{a}-\gamma_{b} \varepsilon_{b}
$$

\section{The closures}

i) We first assume that the mixing pressure tensor $\rho \mathbf{V V} c(1-c)$ reduces to a scalar one; that is to say in the r.h.s. of equation (10) we set $-\nabla(2 \rho K)$.

ii) Secondly, we assume that there exists an increasing function $\Psi$ of the variable $c$ satisfying

$$
\Psi(1)=\gamma_{a}, \quad \Psi(0)=-\gamma_{b}
$$

with the following approximation for the two terms $\mathcal{G}$ and $\mathcal{A}$

$$
\begin{gathered}
\mathcal{G}=\gamma_{a} \varepsilon_{a}-\gamma_{b} \varepsilon_{b} \simeq \varepsilon \Psi(c) \\
\mathcal{A}=\frac{1}{\rho}\left(\frac{1}{c} \nabla P_{a}-\frac{1}{(1-c)} \nabla P_{b}\right) \simeq \varepsilon \nabla \Psi(c)
\end{gathered}
$$

iii) We now make the crucial assumption that the friction coefficient $\sigma$ is large enough if compared to the inverse of the characteristic evolution time. Thus, in equation (12), we withdraw all the terms but the two last ones, so it leads to the following approximation

$$
\mathbf{V} \simeq-\frac{\varepsilon}{\sigma} \nabla \Psi(c)=-D \nabla c, \quad \text { with } \quad D=\frac{\varepsilon}{\sigma} \Psi^{\prime} .
$$

Remark 1. The closure for $\mathcal{A}$ may be justified by the following way. Near $c=1$, if the flow is assumed to be isentropic, we have $P_{a}=P_{r}(c \rho)^{\gamma_{a}} / \rho_{r}^{\gamma_{a}}$ and $P_{r}, \rho_{r}$ constant; so we may state $\frac{1}{c \rho} \nabla P_{a}=\gamma_{a} \frac{P_{r}}{\rho_{r}^{\gamma a}} \rho^{\gamma_{a}-1} c^{\gamma_{a}-2} \nabla c+\gamma_{a} \frac{P_{r}}{\rho_{r} a} c^{\gamma_{a}-1} \rho^{\gamma_{a}-2} \nabla \rho$; then after withdrawing the second term and setting $\rho^{\gamma_{a}-1} \simeq \rho_{r}^{\gamma_{a}-1}$, we get $\mathcal{A} \simeq \frac{1}{c \rho} \nabla P_{a} \simeq \varepsilon \nabla \Psi(c)$ with $\Psi^{\prime}(1)=\gamma_{a}\left(\gamma_{a}-1\right)$. In the case where $\gamma_{a}=\gamma_{b}=\gamma$, the simplest choice for a odd function $\Psi$ satisfying (13) and this last condition is

$$
\Psi(c)=\gamma\left(\frac{7-\gamma}{2}\left(c-\frac{1}{2}\right)+2(\gamma-3)\left(c-\frac{1}{2}\right)^{3}\right) .
$$

Otherwise, we may choose $\Psi(c)=\bar{\gamma}\left(\frac{7-\bar{\gamma}}{2}\left(c-\frac{1}{2}\right)+2(\bar{\gamma}-3)\left(c-\frac{1}{2}\right)^{3}\right)+\left(\gamma_{a}-\gamma_{b}\right) / 2$, where $\bar{\gamma}=$ $\left(\gamma_{a}+\gamma_{b}\right) / 2$.

Remark 2. If the flows for both species are assumed to be isentropic, then the closure for $\mathcal{A}$ comes from the closure for $\mathcal{G}$, after withdrawing the gradient of $\varepsilon$.

According to the approximation of $\mathcal{G}$ and the relation (8), we get

$$
\varepsilon_{a}=\varepsilon \frac{\gamma_{b}+(1-c) \Psi}{c \gamma_{b}+(1-c) \gamma_{a}}, \quad \varepsilon_{b}=\varepsilon \frac{\gamma_{a}-c \Psi}{c \gamma_{b}+(1-c) \gamma_{a}}
$$


If the polytropic coefficients $\gamma_{a}$ and $\gamma_{b}$ are equal for the two ion species, we have $P_{0}=\rho(\gamma-1) \varepsilon$. Otherwise, we may give an equation of state for the averaged fluid. Indeed, according to (14), we get the following relation $P_{0}=\rho(\Gamma(c)-1) \varepsilon$, with $\Gamma(c)=\left(\gamma_{a} \gamma_{b}+c(1-c)\left(\gamma_{a}-\gamma_{b}\right) \Psi(c)\right) /\left(c \gamma_{b}+(1-c) \gamma_{a}\right)$; it is a good equation of state for the averaged fluid, since $\Gamma(c)-1$ is strictly positive.

Now, using the previous closures for $\mathcal{A}$ and $\mathbf{V}$, the evolution equation for concentration and for the total energy reads as

$$
\begin{gathered}
\frac{\partial}{\partial t}(\rho c)+\nabla \cdot(\rho c \mathbf{U})-\nabla \cdot(\rho c(1-c) D \nabla c)=0 \\
\frac{\partial}{\partial t} \mathcal{E}_{0}+\nabla \cdot\left(\mathcal{E}_{0} \mathbf{U}\right)+\nabla \cdot\left(\left(P_{0}+2 \rho K\right) \mathbf{U}\right)-\nabla \cdot[(c(1-c) \Psi \rho \varepsilon-(2 c-1) K) \rho D \nabla c]=0
\end{gathered}
$$

and the velocity equation reads as

$$
\frac{\partial}{\partial t}(\rho \mathbf{U})+\nabla \cdot(\rho \mathbf{U U})+\nabla\left(P_{0}+2 \rho K\right)=0
$$

Now, to get a full model, it suffices to determine the mixing kinetic energy $K$. Of course, one could claim that $K$ has to be equal to expression $c(1-c) D^{2}|\nabla c|^{2} / 2$, but this leads to complicate balance equation for the internal energy. Thus, we prefer to use a simple form for the internal energy balance and to deduce an evolution equation to determine $K$ given by $\mathcal{E}_{0} \rho^{-1}-\varepsilon-\frac{1}{2}|\mathbf{U}|^{2}$.

Notice that according to initial equations (2)(3) and the previous closures, the balance equation for the internal energy $\rho_{a} \varepsilon_{a}+\rho_{b} \varepsilon_{b}$ would read

$$
\frac{\partial}{\partial t}(\rho \varepsilon)+\nabla \cdot(\rho \varepsilon \mathbf{U})+P_{0} \nabla \cdot \mathbf{U}=-\Psi \nabla \cdot(\rho c(1-c) \varepsilon D \nabla c)+2 \sigma \rho K .
$$

But, we prefer to set simply

$$
\frac{\partial}{\partial t} \rho \varepsilon+\nabla \cdot(\rho \varepsilon \mathbf{U})+P_{0} \nabla \cdot \mathbf{U}=2 \sigma \rho K .
$$

[which is justified when $\sigma K$ is large compared to $\nabla(c(1-c) \varepsilon D \nabla c)$. ]

Then, from (15) one gets a coherent evolution equation for $K$ which is

$$
\frac{\partial}{\partial t}(\rho K)+\nabla \cdot(\rho K \mathbf{U})+2 \rho K \nabla \cdot \mathbf{U}+\nabla((2 c-1) \rho K D \nabla c)=\nabla \cdot(\rho c(1-c) \Psi \varepsilon D \nabla c)-2 \sigma \rho K .
$$

But since $\varepsilon \Psi^{\prime}=\sigma D$ we may use the following relation

$$
\nabla \cdot(\rho c(1-c) \Psi \varepsilon D \nabla c)=\sigma \rho c(1-c) D^{2}|\nabla c|^{2}+\Psi \nabla \cdot(\rho c(1-c) \varepsilon D \nabla c)
$$

Therefore, using the Lagrangian derivative $D_{t}=\frac{\partial}{\partial t}+\mathbf{U} . \nabla$, we may summarize the system which we address besides the continuity equation (9)

$$
\begin{gathered}
\rho D_{t} \mathbf{U}+\nabla\left(P_{0}+2 \rho K\right)=0 \\
\rho D_{t} \varepsilon+P_{0} \nabla \cdot \mathbf{U}=2 \sigma \rho K \\
\rho D_{t} c-\nabla \cdot(\rho c(1-c) D \nabla c)=0
\end{gathered}
$$

$$
\rho D_{t} K+2 \rho K \nabla \cdot \mathbf{U}+\nabla((2 c-1) \rho K D \nabla c)-\Psi \nabla \cdot(\rho c(1-c) \varepsilon D \nabla c)=\sigma \rho\left(c(1-c) D^{2}|\nabla c|^{2}-2 K\right)
$$

Of course, there is an energy balance relation associated to this system which is (15). Notice that when coefficient $\sigma$ goes to infinity, coefficient $D$ goes to 0 then this system reduces to the 
classical Euler equations for two fluids without mixing ; indeed if $D$ would be set to zero and if the initial value of $K$ would be zero, then $K(t,$.$) is always equal to 0$.

In equation (19), one sees that when coefficient $1 / \sigma$ is small if compared to the characteristic time of evolution of the plasma, then $K$ will be close to the quanity $c(1-c) D^{2}|\nabla c|^{2} / 2$.

Remark 3. If the initial value $c^{i n}$ of $c$ is a Heavyside function, then the function $c^{i n}$ remains a solution of equation (18), but it is unstable solution. On the other hand, it may be proved easily that for any bounded regular function $D$ of $x$, the solution of (18) remains positive.

Remark 4 This diffusion concentration equation (18) has a form analogous to the one written in [11], (chap. VI, but in this last one, there are a pure diffusion term and a thermo-diffusion term). This kind of equation is also introduced in turbulence models, in motor combustion or in classical model for plasmas (see [12] , [14] for instance). It may be also compared to the one introduced [3] (§9.5). In this last case (besides a thermo-diffusion term), one can find an equation which reads with our notations

$$
\rho D_{t} c-\nabla \cdot\left(\rho D_{0} \nabla c\right)=0
$$

with $D_{0}$ depending on the temperature and on the plasma components but independent of $c$. Notice that if one wants to follow the expansion of a mixing domain with a model like (20), the relative velocity becomes

$$
\mathbf{V}=-\frac{1}{c(1-c)} D_{0} \nabla c=-D_{0} \nabla\left(\log \frac{c}{1-c}\right)
$$

which is infinite near the front of the mixing domain (when $c$ tends to 0 or 1 ). Our model overcomes this difficulty and may be interpreted as a limitation of the mass flux at the boundary of the mixing model. In a certain way, we have changed the unbounded function $\log \frac{c}{1-c}$ by a bounded one $\psi(c)$.

Remark 5. For modeling the mixing, we use the formula given by [3] (p. 119) and we assume that $\gamma_{a}=\gamma_{b}=5 / 3$. Then we get

$$
\sigma=\rho \beta_{0}\left(\left(\frac{9 \pi}{2 \alpha_{0}^{2}}\right)^{1 / 3} \frac{2}{3}\left(\varepsilon_{a}+\varepsilon_{b}\right)+V_{r}^{2}\right)^{-3 / 2},
$$

with

$$
\beta_{0}=4 \pi \eta_{a}^{2} \eta_{b}^{2}\left(m_{a}+m_{b}\right) q_{e}^{4} \log \Lambda_{a b}
$$

where $q_{e}$ is the electron charge, $\log \Lambda_{a b}$ a Coulomb logarithm (which is in the order of some units), $\alpha_{0}^{2}$ is a dimensionless coefficient in the order of 1 depending on the plasma and $V_{r}$ is a corrector term in the order of the relative velocity. Then, by using the expressions of $\varepsilon_{a}$ and $\varepsilon_{b}$ (see (14)), $D$ has the following form

$$
D(c) \simeq \frac{\varepsilon}{\beta_{0}} \frac{\Psi^{\prime}(c)}{\rho}\left(\left(\frac{9 \pi}{2 \alpha_{0}^{2}}\right)^{1 / 3}\left(\frac{4}{3}+(2-4 c) \frac{\Psi(c)}{5}\right) \varepsilon+V_{r}^{2}\right)^{3 / 2} .
$$

One has to evaluate the corrector term $V_{r}^{2}$, but it is possible to check that this added term is not negligible only when the ion temperature is not large, that is to say at the beginning of mixing phenomenon, so it may be seen that a very crude evaluation of this term is sufficient. As a matter of fact, we set

$$
V_{r}^{2}=\varepsilon^{5} /\left(L \rho \beta_{0}\right)^{2}
$$

where $L$ is a constant which is a characteristic value of the width of the mixing zone (it depends on the actual cases and in our hot plasma cases it is set to some micrometers).

According to the fact that $|\Psi(c)| \leq 5 / 3$, we can see that $D(c) \geq C \frac{\varepsilon^{5 / 2}}{\rho \beta_{0}}$ for some constant $C$. 


\subsection{Model accounting for the electron temperature}

This technical section is devoted to the statement of the extension of the the previous mixing model, when we take into account the coupling between the ion and electron temperatures (as described in section 1.2). For the sake of simplicity, we assume here that $\gamma_{g}=\gamma_{l}=\gamma$. Recall that

$$
N_{e}=\rho\left(c \eta_{a}+(1-c) \eta_{b}\right) \text {. }
$$

Let us first evaluate the quantity $\Upsilon_{0}=\Upsilon_{a}+\Upsilon_{b}$, we get

$$
\Upsilon_{0}=\rho^{2}\left(c \eta_{a}+(1-c) \eta_{b}\right)\left[\left(c \nu_{a}^{E}+(1-c) \nu_{b}^{E}\right) T_{e}-c \nu_{a}^{E} \frac{\varepsilon_{a}}{C_{v, a}}-(1-c) \nu_{b}^{E} \frac{\varepsilon_{b}}{C_{v, b}}\right] .
$$

Recall that according to the closure we have made, the relations (14) express $\varepsilon_{a}$ and $\varepsilon_{b}$ with the help of $\varepsilon$; therefore, it leads to

$$
\Upsilon_{0}=\rho^{2}\left(c \eta_{a}+(1-c) \eta_{b}\right)\left(c \nu_{a}^{E}+(1-c) \nu_{b}^{E}\right)\left(T_{e}-\frac{\varepsilon}{C_{v, 0}}\right)
$$

where $C_{v, 0}$ is given by

$$
\frac{c \nu_{a}^{E}+(1-c) \nu_{b}^{E}}{C_{v, 0}}=\frac{\nu_{a}^{E} c(\gamma+(1-c) \Psi)}{\gamma C_{v, a}}+\frac{\nu_{b}^{E}(1-c)(\gamma-c \Psi)}{\gamma C_{v, b}} .
$$

Thus, quantity $\Upsilon_{0}$ has a classical form $\Omega\left(T_{e}-\varepsilon / C_{v, 0}\right)$, with $\Omega$ positive.

Now, defining $\zeta=\left(\eta_{a}-\eta_{b}\right) /\left(c \eta_{a}+(1-c) \eta_{b}\right)$, we get a modified expression for the ion energy balance

$$
\begin{aligned}
& \rho D_{t}\left(\frac{\mathcal{E}_{0}}{\rho}\right)+\nabla \cdot\left(P_{0} \mathbf{U}+\right.2 \rho K \mathbf{U})+\mathbf{U} \cdot \nabla P_{e}-\nabla \cdot((c(1-c) \Psi \rho \varepsilon-(2 c-1) K) \rho D \nabla c)= \\
& \Upsilon_{0}-\zeta c(1-c) D \nabla c . \nabla P_{e}
\end{aligned}
$$

Moreover, for the electron energy, equation (6) reads by a classical way and the full model becomes

$$
\begin{gathered}
\rho D_{t}\left(\rho^{-1}\right)-\nabla \cdot \mathbf{U}=0 \\
\rho D_{t} \mathbf{U}+\nabla\left(P_{0}+P_{e}+2 \rho K\right)=0, \\
\rho D_{t} \varepsilon+P_{0} \nabla \cdot \mathbf{U}=2 \sigma \rho K+\Upsilon_{0}, \\
\rho D_{t} c-\nabla \cdot(\rho c(1-c) D \nabla c)=0, \\
\rho D_{t} K+2 \rho K \nabla \cdot \mathbf{U}+\nabla((2 c-1) \rho K D \nabla c)=\Psi \nabla \cdot(c(1-c) \rho \varepsilon D \nabla c) \\
\quad+\sigma \rho\left[c(1-c) D^{2}|\nabla c|^{2}-2 K\right]+\zeta c(1-c) D \nabla c \cdot \nabla P_{e} \\
\rho D_{t}\left(\mathcal{E}_{e} / \rho\right)+P_{e} \nabla \cdot \mathbf{U}-\nabla \cdot\left(\mathcal{E}_{e} c(1-c) \zeta D \nabla c\right)-P_{e} \nabla \cdot(c(1-c) \zeta D \nabla c)+\nabla \cdot \mathbf{q}_{\mathrm{th}}=-\Upsilon_{0} .
\end{gathered}
$$

Remark The structure of these three energy equations is satisfying because we can observe the coupling terms $2 \rho \sigma K, 2 \rho^{2} \nu_{e}^{0} K$ and $\Upsilon_{0}$ appear with opposite signs in the right hand side of these equations. On the other hand, one can check that the global energy $\mathcal{E}_{0}+\mathcal{E}_{e}$ satisfies a conservative balance equation.

\section{$3 \quad$ Numerical issues}

The aim of this work was the implementation of the previous model in a $2 \mathrm{D}$ multi-material hydrodynamics code for plasma flow. Without going into the details of the numerical methods for the simulations without the mixing model, we only recall briefly that for the hydrodynamics part of the model, one uses a Wilkins scheme which is based on a staggered grid method; that is to say, the density, the energy, the pressures and the concentration are evaluated in the center of each cell and the velocity at the cell vertices (in a one-dimension framework, it reduces to the classical Von Neuman-Richtmyer scheme, cf. [13]). 


\subsection{Some remarks on the numerical methods}

At each time step, there are two stages. At the first stage of the leapfrog method, one updates the velocity and one moves each node with its velocity. At the second stage, one updates the density and one solves the internal energy equation.

The modifications due to the mixing model are the following. The mixing kinetic energy $K$ and the concentration $c$ are evaluated at the center of each cell. First one has to deal with the concentration equation (18): we make a time implicit discretization of the diffusion term

$$
\frac{\partial}{\partial x}\left(c(1-c) D \frac{\partial}{\partial x} c\right)
$$

the key point is the evaluation of the mean value of $[c(1-c) D]_{i+1 / 2}$ on each interface $i+1 / 2$ between the cells $i$ and $i+1$. We state in the one-dimension framework

$$
\left[c(1-c) D \frac{\partial c}{\partial x}\right]_{i+1 / 2}=2 \frac{c_{i+1}-c_{i}}{\left(\delta x_{i+1}+\delta x_{i}\right)}\left(c_{i+1}\left(1-c_{i+1}\right) D_{i+1}+c_{i}\left(1-c_{i}\right) D_{i}\right),
$$

where the $c_{i}$ are the unknown values; we have the analogous for the two-dimension framework. So at each time step, one has to solve a non linear matrix system by an iterative method.

Then, using the value of the normal relative velocity $-D \mathbf{n} . \nabla c$ at the interfaces between two neighbouring cells ( $\mathbf{n}$ is the unit normal to the interface), one solves the mixing kinetic energy equation (28). It is done with a finite volume scheme; the only technical point is the advection term $(2 c-1) \rho K D \nabla c$ which is handled with an upwind technique which is explicit with respect to the time (in each cell, it depends on the sign of $(2 c-1) \mathbf{n} . \nabla c$ in the neighbouring cells). Of course, the time step has to satisfy a classical stabilty criterium for this upwind technique which is of the type $|(2 c-1) D \mathbf{n} . \nabla c| \delta t / \delta x \leq 1$, but this criterium is generally less strong than the standard one related to the Wilkins scheme $v_{\text {ther }} \delta t / \delta x \leq 1$, where $v_{\text {ther }}$ is the sound speed.

Moreover, for the ion internal energy equation (26), one has to take into account extra terms in the right hand side : no difficulty for the term $2 \sigma \rho K$ and the coupling term $\Upsilon_{0}$ which reads as $\Omega\left(T_{e}-\varepsilon / C_{v, 0}\right)$ is handled classically by implicit evaluation of $T_{e}$ and $\varepsilon$.

\subsection{Numerical results}

\section{a. One-dimension toy problem.}

In a first stage, we consider here a toy one-dimension problem and we wish to compare the numerical results obtained with the proposed model and the ones obtained with a multifluid code which handles a model of the type of [3], (cf. MULTIF code [5]). We consider here a simple case, that is to say without laser and without radiative loss: there are two slabs occupied initially by a pure gold plasma which are separated by vacuum. Both fluids are characterized by the same polytropic coefficient $\gamma$ (equal to $\frac{5}{3}$ ) and the same heat capacity coefficient; their initial densities are equal to $0.0029 \mathrm{~g} / \mathrm{cm}^{3}$ and their initial temperatures equal to $1 \mathrm{keV}$. The initial velocities are $9.510^{7} \mathrm{~cm} / \mathrm{s}$ for the left fluid and $-9.510^{7} \mathrm{~cm} / \mathrm{s}$ for the right one. For the evaluation of $D$ defined by (21), we take $\alpha_{0}=0.84$ (since the two fluids are identical [3]) and $\beta_{0}=2.2210^{36}$ (in CGS units) which corresponds to $\log \Lambda_{a b}=5$ (a mean value of the Coulomb logarithm in the mixing region during the time of simulation).

With these values, the slowing down distance of the ions on the other ion population in the mixing region is in the order of $130 \mu \mathrm{m}$ and the corresponding slowing down time can reach 160 ps.

The spatial grid is $1000 \mu \mathrm{m}$ long and composed of about 1000 meshes. As the slowing down distance represents several meshes, a strong collision with mixing between the two fluids occurs. The ionization level fixed to approximately 58 , that is to say the electron equation of state is

$$
\mathcal{E}_{e}=\frac{3}{2} 58 \rho T_{e}
$$




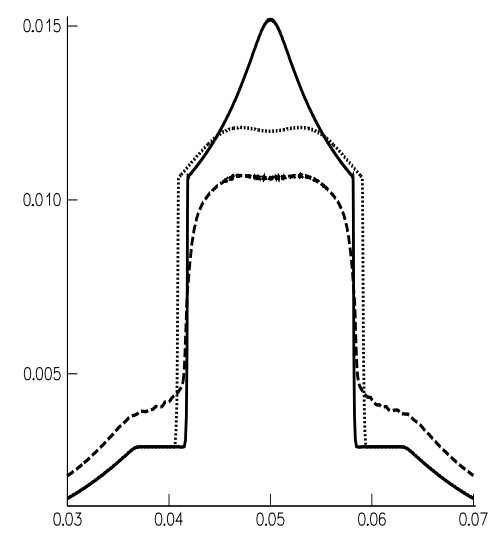

Figure 1: Profile of the density at time $t=300 \mathrm{ps}$, without the mixing model (solid line), with the mixing model (doted line), compared to the result given the MULTIF code (the lowest dashed line).

For the closure function $\Psi$, we have chosen as above. Moreover, in the above mentioned Spitzer formula for the electron thermal flux, we take $\kappa\left(T_{e}\right)=k(x) T_{e}^{5 / 2}$, where $k(x)$ is a function depending weakly on the space variable (due to the so-called electron Coulomb logarithm).

We show on figure 1, the density profiles at $300 \mathrm{ps}$, in the standard case without accounting for the mixing model and the same density profiles in the case where the mixing model is taken into account by using the above value of $\sigma_{0}$. Notice that $300 \mathrm{ps}$ corresponds to a few relaxation times. We can observe that the level of the maximum of the density is about $0.015 \mathrm{~g} / \mathrm{cm}^{3}$ without the mixing model but only $0.012 \mathrm{~g} / \mathrm{cm}^{3}$ with the mixing model, it is closer to the reference value which is $0.011 \mathrm{~g} / \mathrm{cm}^{3}$ given by the MULTIF code.

Notice that in the simulations with the mixing model, we have to give an ad hoc value for the length $L$, in the formula defining the corrector term $V_{r}^{2}$; but we may check that if we change this value by a factor 2 , the above values of density do not vary by more than $5 \%$. Moreover, choosing other functions for $\Psi$ (satisfying (13)) leads also to small variations for these values of density.

\section{Two dimension simulations}

In this simulation, we have two gold diskes which are separated by vacuum. Each disk is heated by some laser beams; it is expanded and a collision between the two diskes occurs. We have performed numerical simulations with the two-dimension axi-symmetric code FCI2 [1] with an arbitrary Lagrange-Euler framework accounting for laser heating (by a ray-tracing method) and electron thermal conduction. We have compared the results of a simulation made without mixing model and another one accounting for mixing model from the time $1.9 \mathrm{~ns}$ (which is the time of collision between the two diskes).

Thus, on figure 2, one may compare the density without and with mixing model at time $t=2 \mathrm{~ns}$.

Moreover, on figure 3, one compares firstly the ion temperature without and with mixing model at time $t=2 n s$ and secondly, on figure 4 , the map of electron temperature at the same time.

It may be noticed that the ion temperature quickly increases up to $15010^{6} \mathrm{~K}$ in the collision region with or without mixing. This is due to the fact that almost all the initial kinetic energy of the two fluids is very quickly converted into internal energy.

\section{Conclusion}

We have proposed a model for mixing of two plasmas; it has been extended easily to the framework of the two-temperature Euler system. The underlying assumption is that the relative velocity between the two fluids is small compared to the sound speed of the flow. This model consists in coupling the classical Euler equations with a concentration equation (which is a non linear 

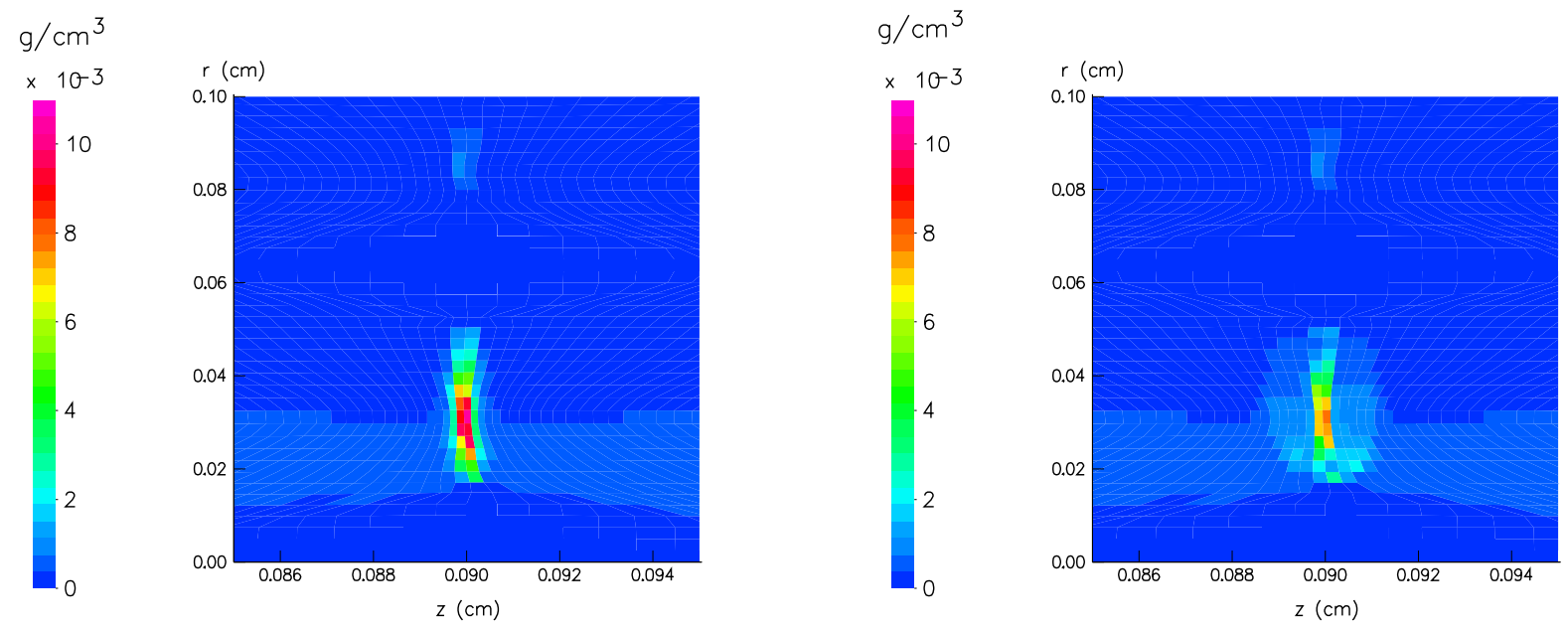

Figure 2: Map of the density at 2. ns, without (on the left) and with the mixing model (on the right).
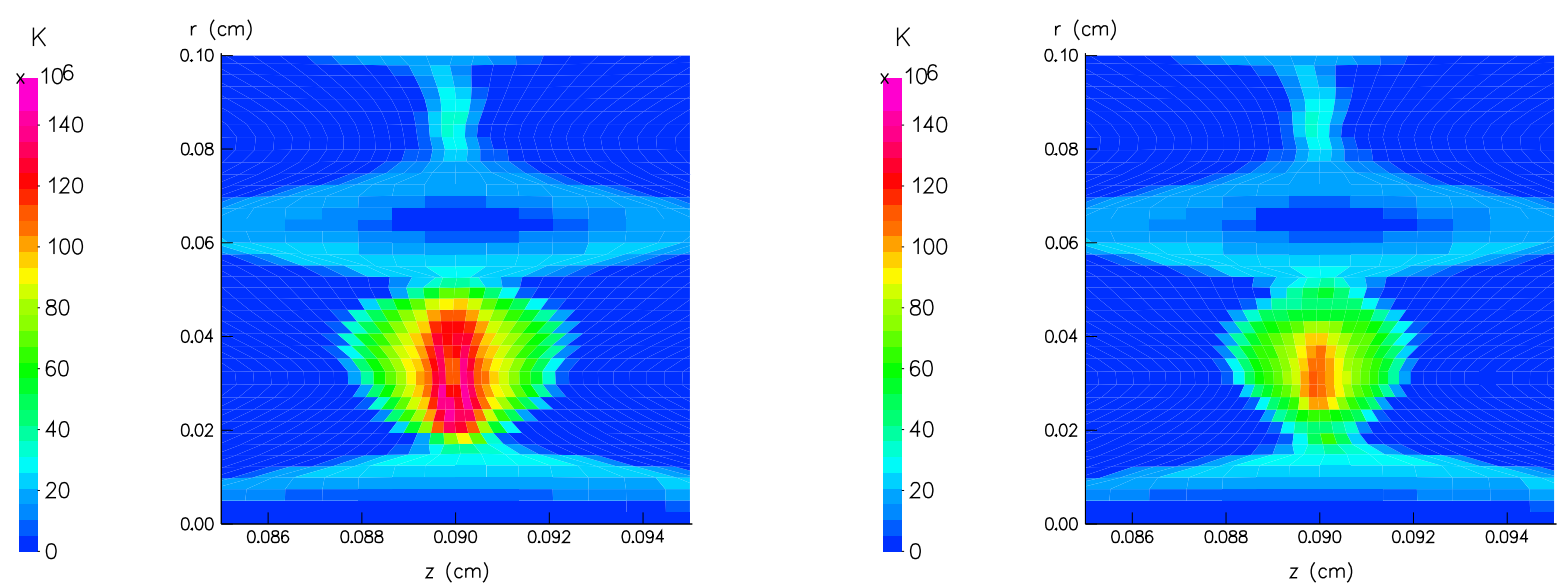

Figure 3: Map of the ion temperature at 2. ns, without (on the left) and with the mixing model (on the right). 

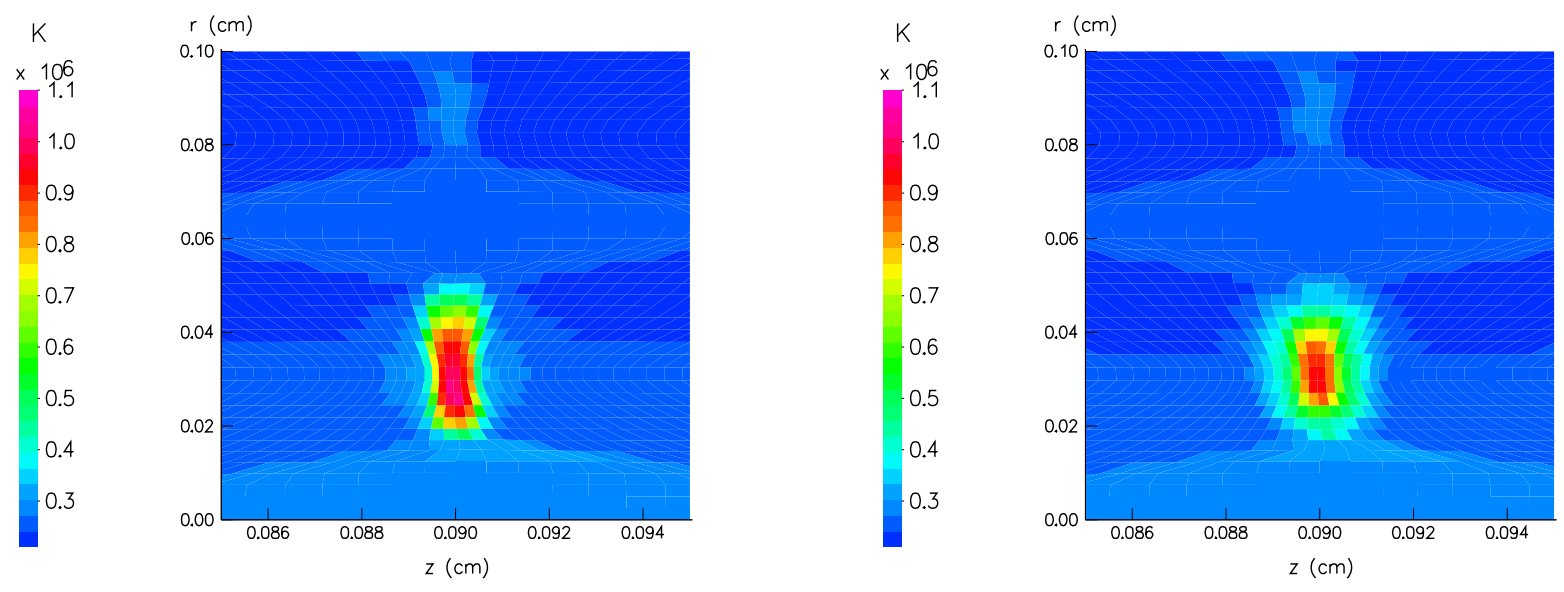

Figure 4: Map of the electron temperature at 2. ns, without (on the left) and with the mixing model (on the right).

diffusion equation) and an evolution equation for the mixing kinetic energy $K$. The implementation of this model has been performed in a two-dimension Arbitrary-Lagange-Euler code where electron thermal conduction, radiative conduction and laser enegy deposition are taken into account.

Without the mixing model, when a strong collision between the two fluids occurs, a bump of the density $\rho$ may be observed in the collision region. Accounting the mixing model, the numerical results show a decrease of the level of this bump; this leads to a notable decrease of the radiative emission which is, roughly speaking, proportional to the square of $\rho$. Notice that our model may be also generalized to mixing accounting for three fluids, using two concentration diffusion equations. 


\section{References}

[1] E. Buresi et al., Laser Program Development at CELV. Laser Part. Beams 4, p. 531 (1986).

[2] R. L. Bowers and J.R. Wilson, Numerical Modeling in Applied Physics, Jones-Bartlett, Boston (1991).

[3] A. Decoster, Modeling of collisions, P.A. Raviart ed., Masson, Paris (1997).

[4] R. Dautray, J. Watteau, La Fusion par Confinement Inertiel, tome II, Eyrolles, Paris (1992).

[5] C. Chenais-Popovics, O. Larroche et al., Kinetic to thermal energy transfer and interpenetration in the collision of laser-produced plasmas, Phys. Plasmas 4, p. 190 (1997).

[6] A. J. Scannapieco, B. Cheng, A multifluid interpenetration mix model. Physics Letters A, 299, p. 49 (2002).

[7] H.B. Stewart, B. Wendroff, Two-phase Flows : Models and methods, J. Comp. Phys. 56, p. 363 (1984).

[8] M. Ishii, Thermo-Fluid dynamic theory of two phase flows, Eyrolles, Paris (1975).

[9] B. Desprès, Lagrangian system of conservation laws, Numerische Math. 89, p. 99 (2001).

[10] A.D. Resnyansky, B.E. Milton, E.I. Romansky. A two-phase Shock-wave model, J. S. M. E. Int. Conf. on Fluid Engineering, Tokyo (1997).

[11] L. Landau, E. Lifchitz, Fluids Dynamics, MIR edition, Moscow (1954).

[12] S.P. Hirshman, Transport of a multiple-ion species plasma, preprint.

[13] R.D. Richtmyer, K.W. Morton, Difference methods for initial value problems, J. Wiley (1967).

[14] D.L. Youngs. Numerical simulations of turbulent mixing, Physica D, 12, p. 32 (1984).

[15] D. C. Wilson, A. J. Scannapieco, Degradation of radiatively driven ICF capsula. Physics of Plasmas, 10, p. 4427 (2003) 